Pacific Journal of Mathematics

THE TYPESET AND COTYPESET OF A RANK 2 ABELIAN 


\title{
THE TYPESET AND COTYPESET OF A RANK 2 ABELIAN GROUP
}

\author{
PhILLIP SCHULTZ
}

Let $T$ and $T^{\prime}$ be sets of types. This paper describes necessary and sufficient conditions on $\left(T, T^{\prime}\right)$ for the existence of a rank 2 torsion-free abelian group $A$ such that $T$ is the set of types of elements of $A$, and $T^{\prime}$ is the set of types of rank 1 factor groups of $A$. Moreover, it classifies all such $A$ and gives necessary and sufficient conditions for $A$ to be completely anisotropic.

1. Introduction. This paper describes those pairs $\left(T, T^{\prime}\right)$ of sets of types such that for some reduced rank 2 torsion-free abelian group $A, T$, the typeset, is the set of types of elements of $A$, and $T^{\prime}$, the cotypeset, is the set of types of rank 1 torsion-free factor groups of $A$.

This completes a line of research initiated by Beaumont and Pierce [1], to which notable contributions were made by Koehler [6], Dubois [2] and [3], and Ito [5]. However, the methods of this paper, unlike those cited above, can be extended to groups of arbitrary finite rank, using the inductive technique of [7].

Apart from its use in the construction of the group $A$, the advantages of introducing the pair $\left(T, T^{\prime}\right)$ are twofold: firstly it provides a finer classification of rank 2 groups than the typeset alone; and secondly in the case of groups of arbitrary finite rank it provides a useful classification scheme for the torsion theory generated by the group $A$. The details will appear elsewhere, but roughly the idea is the following: for any abelian group $A$, the torsion-free class of the torsion theory generated by $A$ is

$$
A^{\perp}=\{X: X \text { is torsion-free and } \operatorname{Hom}[A, X]=0\} .
$$

Let $\left(T, T^{\prime}\right)$ be the typeset and cotypeset of a torsion-free group $A$, let $C$ be any completely decomposable group with typeset $T$, and $D$ any completely decomposable group with typeset $T^{\prime}$. Then

$$
C^{\perp} \subseteq A^{\perp} \cong D^{\perp} .
$$

This yields a classification of torsion theories in terms of completely decomposable groups.

Section 2 of this paper compares and shows the essential equivalence of the structure theories of [1] and [7], and $\S 3$ establishes several invariants of rank 2 groups. These invariants are used in $\S 4$ to develop necessary conditions on $\left(T, T^{\prime}\right)$. The computations which prove that these conditions are also sufficient comprise $\S 5$, 
while $\S 6$ is a proof that the group constructed does in fact realize $\left(T, T^{\prime}\right)$. The construction of $\S 5$ is analyzed in $\S 7$ and the number of groups realizing $\left(T, T^{\prime}\right)$ is counted. Finally in $\S 8$ the completely anisotropic groups realizing $\left(T, T^{\prime}\right)$ are constructed.

Throughout we employ the standard notation of [4] except where otherwise noted. One major exception is the following:

A height is a function $h$ from the set $\boldsymbol{P}$ of primes into $N \cup\{\infty\}$. A type is an equivalence class of heights with respect to the usual equivalence relation $k \sim h$ if $\sum_{p \in P}|h(p)-k(p)|<\infty$. The height of an element $x$ of a group $A$ is denoted $h_{A}(x)$ or $h(x)$ if no ambiguity results. A generalized height is a function from $\boldsymbol{P}$ into $\boldsymbol{Z} \cup\{\infty\}$; for example, if $r=a / b$ is rational, then $h(r)$ is the generalized height defined by $h(r)(p)=h_{z}(a)(p)-h_{z}(b)(p)$.

We frequently use the ring $\hat{Z}$, the closure in the $n$-adic topology of the ring $\boldsymbol{Z}$ of integers. However we are interested only in its algebraic structure: $\hat{\boldsymbol{Z}}=\prod_{p \in P} \boldsymbol{Z}_{p}$, where $\boldsymbol{Z}_{p}$ is the ring of $p$-adic integers.

An arrow $\succ$ represents a monomorphism, $\rightarrow$ an epimorphism.

2. The structure theories of [1] and [7]. Proofs of the following assertions are in $\S 3$ of [7].

Let $A$ be a reduced rank 2 torsion-free group, and let $\mathrm{a} \mapsto 1 \otimes a$ be the canonical embedding of $A$ into its divisible hull $V=Q \otimes A$. For any $x \in A$, let $W(x)$ be the pure subgroup generated by $x$. Suppose $\{x, y\}$ is a basis of $A$; then $A /(W(x) \oplus W(y))$ is isomorphic to $S=$ $\bigoplus_{p \in P} \boldsymbol{Z}\left(p^{k(p)}\right), 0 \leqq k(p) \leqq \infty$.

$V$ contains independent subgroups $\bar{W}(x), \bar{W}(y)$ containing $y, x$ respectively, such that $\bar{W}(x) \cong A / W(x), \bar{W}(y) \cong A / W(y)$ and $k(p)=$ $h_{\bar{W}(x)}(y)(p)-h_{A}(y)(p)=h_{\bar{W}(y)}(x)(p)-h_{A}(x)(p)$. There exists a cartesian square:

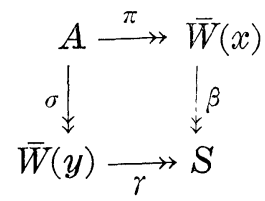

in which $A=\{r x+s y: r, s \in \boldsymbol{Q}, \beta(s y)=\gamma(r x)\}$

$$
\begin{aligned}
& \pi(r x+s y)=s y ; \sigma(r x+s y)=r x \\
& \operatorname{ker} \beta=\operatorname{ker} \sigma=W(y) ; \operatorname{ker} \gamma=\operatorname{ker} \pi=W(x) .
\end{aligned}
$$

The pair $(\beta, \gamma)$ of epimorphisms may be replaced by a pair $\left(\beta^{\prime}, \gamma^{\prime}\right)$ provided $(\beta, \gamma)$ induces the same automorphism of $S$ as does $\left(\beta^{\prime}, \gamma^{\prime}\right)$. In this case, we write $(\beta, \gamma) \sim\left(\beta^{\prime}, \gamma^{\prime}\right)$ and denote the equivalence class by $[\beta, \gamma]$. 
Conversely, let $\{x, y\}$ be a basis of a rational vector space $V$; let $S$ be any subgroup of $\boldsymbol{Q} / Z$; let $\bar{W}(x), \bar{W}(y)$ be independent subgroups of $V$ containing $y, x$ respectively, and let $\beta: \bar{W}(x) \rightarrow S$ and $\gamma: \bar{W}(y) \rightarrow S$ be epimorphisms. Then the pullback $A$ of $(\beta, \gamma)$ is a rank 2 torsionfree group containing independent pure subgroups $W(x)=\operatorname{ker} \gamma, W(y)=$ ker $\beta$ such that $x \in W(x), y \in W(y)$, and the diagram above is commutative. If $\left(\beta^{\prime}, \gamma^{\prime}\right)$ is another pair of such epimorphisms, with pullback $A^{\prime}$, then $A^{\prime}=A$ iff $(\beta, \gamma) \sim\left(\beta^{\prime}, \gamma^{\prime}\right)$. Furthermore, $A$ is quasi-isomorphic to $A^{\prime}$, denoted $A \doteq A^{\prime}$, iff the automorphism of $S$ induced by $(\beta, \gamma)$ differs from the automorphism induced by $\left(\beta^{\prime}, \gamma^{\prime}\right)$ by a rational multiple. We denote this construction by $\langle A, W(x), W(y), \bar{W}(x), \bar{W}(y)$, $S,[\beta, \gamma]\rangle$.

The details and proofs of the previous two paragraphs in [7] deal with the more general case in which $V$ has arbitrary dimension. It is stated in [7] that the rank 2 case is essentially the same as the construction in [1], and since we shall make heavy use of Beaumont and Pierce's results, the connection must now be made clear.

By [1, Theorem 2.10], a reduced rank 2 group $A$ with distinguished basis $\{x, y\}$ determines a unique pair $(\Sigma, X)$, where $\Sigma$ is a height and $X$ an equivalence class of pairs $(\xi, \eta)$ from $\hat{\boldsymbol{Z}} \times \hat{\boldsymbol{Z}}$. A pair $(\xi, \eta)$ is equivalent to a pair $\left(\xi^{\prime}, \eta^{\prime}\right)$ provided that

(i ) $h(\xi)=h\left(\xi^{\prime}\right), h(\eta)=h\left(\eta^{\prime}\right)$

and

(ii) $h\left(\xi \eta^{\prime}-\xi^{\prime} \eta\right) \geqq \Sigma+h(\xi)+h(\eta)$.

(Fuchs prefers multiplicative notation for "addition" of heights and types [4, II, p. 110], but throughout, I shall conform to the additive notation of [1].)

For given $\langle A, x, y\rangle$, the height $\Sigma$ is defined by

$$
A /(W(x) \oplus W(y)) \cong \bigoplus_{p \in P} \boldsymbol{Z}\left(p^{\mathcal{E}(p)}\right),
$$

and a pair $(\xi, \eta)$ is specified by

(i) $h(\xi)=h(y), h(\eta)=h(x)$

(ii) $\forall p \in \boldsymbol{P}$, let $a, b$ be integers with $h(a)(p)=h(y)(p)=u, h(b)(p)=$ $h(x)(p)=v$, and $k$ an integer such that $0 \leqq k \leqq \Sigma(p)$. Then $p^{-\langle k+u+v)}(a x+b y) \in A$ iff $h(a \eta(p)-b \xi(p))(p) \geqq k+u+v$. (This inequality of course is suitably interpreted in case $u$ or $v$ is infinite.) Beaumont and Pierce show that (i) and (ii) define $(\xi, \eta)$ up to equivalence, and conversely, a pair $(\Sigma, X)$ determines $\langle A, x, y\rangle$ up to isomorphism; the structure is denoted $\langle A, x, y\rangle \rightarrow(\Sigma, X)$.

METATHEOREM. The structure theories of [7] (rank 2 case) and [1] are essentially the same. 
Proof. The height $\Sigma$ is defined like the height $k$, and up to isomorphism, $\langle W(x), W(y), \bar{W}(x), \bar{W}(y), S\rangle$ can be recovered from $x, y$, and $\Sigma$, and vice versa. Hence in order to establish the correspondence we must show how $X$ determines $[\beta, \gamma]$ and vice versa.

Suppose $\langle A, x, y\rangle \rightarrow(\Sigma, X)$, and let $(\xi, \eta) \in X$. Let $h(x)(p)=v$, $h(y)(p)=u$. If $v$ or $u$ is infinite, then $\Sigma(p)=\xi(p)=\eta(p)=0$, and any automorphism of $S$ has zero $p$-component. Furthermore, any $\left(\xi^{\prime}, \eta^{\prime}\right)$ from $X$ also has zero $p$-component, so in this case, the $p$-component of the automorphism determined by $[\beta, \gamma]$ is completely determined.

Assume then that $u$ and $v$ are both finite. For any $p$-adic integer $e$ represented in the form $\sum_{i=0}^{\infty} s_{i} p^{i}$, let $e_{j}$ be the $j$ th segment $\sum_{i=0}^{j-1} s_{i} p^{i}$. Define $p$-adic units $c(p), d(p)$ by $c(p)=p^{-u} \xi(p), d(p)=p^{-v} \eta(p)$, and denote their $j$ th segments by $c_{j}(p), d_{j}(p)$. For any integer $j$, $0 \leqq j \leqq \Sigma(p)$, define

$$
\gamma\left(p^{-(j+v)} c_{j}(p) x\right)=\beta\left(p^{-(j+u)} d_{j}(p) y\right) .
$$

This makes sense, since $h\left(p^{u} c_{j}(p) \eta(p)-p^{v} d_{j}(p) \xi(p)\right)(p) \geqq j+u+v$ implies $p^{-(j+v)} c_{j}(p) x+p^{-(j+u)} d_{j}(p) y \in A$.

Now if $j=\Sigma(p)<\infty$, then $\gamma\left(p^{-\langle j+v)} c_{j}(p) x\right)$ is an element of maximal order $p^{j}$ in the cyclic group $S_{p} \cong \boldsymbol{Z}\left(p^{j}\right)$, and similarly $\beta\left(p^{-(j+u)} d_{j}(p) y\right)$ is a generator of $S_{p}$, so the equation $(*)$ determines the $p$-component of the automorphism of $S$ induced by $(\beta, \gamma)$.

If $\sum(p)=\infty,\left\{\gamma\left(p^{-(j+v)} c_{j}(p) x\right): j=1,2, \cdots\right\}$ is a set of generators for $S_{p} \cong Z\left(p^{\infty}\right)$, as is $\left\{\beta\left(p^{-(j+u)} d_{j}(p) y\right): j=1,2, \cdots\right\}$, so the equations (*) completely determine the $p$-component of the automorphism of $S$ induced by $(\beta, \gamma)$.

We have shown that $(\xi, \eta)$ determines a unique class $[\beta, \gamma]$. Suppose that also $\left(\xi^{\prime}, \eta^{\prime}\right) \in X$, and let $c^{\prime}(p), d^{\prime}(p)$ be the corresponding $p$-adic units. Since $h\left(\xi(p) \eta^{\prime}(p)-\xi^{\prime}(p) \eta(p)\right)(p) \geqq \sum(p)+u+v$, $p^{-(j+v)} c_{j}^{\prime}(p) x+p^{-(j+u)} d_{j}^{\prime}(p) y \in A$, so $\beta\left(p^{-(j+v)} c_{j}^{\prime}(p) x\right)=\gamma\left(p^{-(j+u)} d_{j}^{\prime}(p) y\right)$, i.e., $\left(\xi^{\prime}, \eta^{\prime}\right)$ determines the same class $[\beta, \gamma]$ as does $(\xi, \eta)$.

Conversely, suppose given $\langle A, W(x), W(y), \bar{W}(x), \bar{W}(y), S,[\beta, \gamma]\rangle$. Let $\bar{\beta}: \bar{W}(x) / W(y) \rightarrow S, \bar{\gamma}: \bar{W}(y) / W(x) \rightarrow S$ be the isomorphisms induced by $(\beta, \gamma) \in[\beta, \gamma]$. Let $p \in \boldsymbol{P}$ and let $u=h(y)(p), v=h(x)(p)$. If $u$ or $v$ is infinite, $S_{p}=0$, so for any choice of $(\xi, \eta)$ we must have $\xi(p)=0=\eta(p)$. Thus we may assume $u$ and $v$ are finite.

If $k(p)<\infty$, there is a rational $p$-adic unit $c(p)$ such that

$$
\bar{\beta}\left(p^{-(k(p)+u)} c(p) y+W(y)\right)=\bar{\gamma}\left(p^{-(k(p)+v)} x+W(x)\right),
$$

since these elements generate $S_{p} ; c(p)$ is unique modulo $p^{k(p)}$.

If $k(p)=\infty$, there is a unique $p$-adic unit $c(p)$ such that, for all $j=1,2, \cdots$,

$$
\left.\bar{\beta}\left(p^{-(j+u}\right) c_{j}(p) y+W(y)\right)=\bar{\gamma}\left(p^{-(j+v)} x+W(x)\right),
$$


since those elements form a canonical set of generators for $S_{p}$.

Now let $\xi(p)=p^{u} c(p), \eta(p)=p^{v}$ for all $p$, and let $X$ be the equivalence class of $(\xi, \eta)$; from the construction we have $\langle A, x, y\rangle \rightarrow$ $(\Sigma, X)$.

Suppose now we start with $[\beta, \gamma]$ and construct $(\xi, \eta) \in X$ as above. An application of the method of the third paragraph of this section yields $c(p)=p^{-u} \xi(p), d(p)=1$ for all $p$, and hence the original $[\beta, \gamma]$ is recovered.

Conversely, starting with $(\xi, \eta) \in X$ yields a pair $(\beta, \gamma)$ by equations $\left(^{*}\right)$. Now for all primes $p$ and $j \leqq \Sigma(p), d_{j}(p)$ acts as an automorphism on $S_{p}$ such that

$$
\bar{\beta}\left(p^{-(j+u)} c_{j}(p) d_{j}(p)^{-1} y+W(y)\right)=\bar{\gamma}\left(p^{-(j+v)} x+W(x)\right) .
$$

The method above yields $\left(\xi^{\prime}, \eta^{\prime}\right)$, where $\xi^{\prime}(p)=p^{u} c(p) d(p)^{-1}, \eta^{\prime}(p)=p^{v}$, and a short computation shows $\left(\xi^{\prime}, \eta^{\prime}\right) \in X$. Thus applying the constructions consecutively in either order recovers the initial invariants, as was to be proved.

CoROLLARY 1. Given $\langle A, x, y\rangle$, there is a unit $c$ of $\hat{\boldsymbol{Z}}$ such that (a) if $k(p)<\infty, c(p)$ is uniquely determined modulo $p^{k(p)}$ and

$$
p^{-(k(p)+v)} x+p^{-(k(p)+u)} c(p) y \in A \text {, and }
$$

(b) if $k(p)=\infty, c(p)$ is unique and for all $j$,

$$
p^{-(j+v)} x+p^{-(j+u)} c_{j}(p) y \in A .
$$

3. Invariants of rank 2 groups. Having established the correspondence between the two theories, we can use [1] to list some useful invariants of a rank 2 group $A$ in terms of the structure theorem of [7].

Proposition 1. [1, $\$ 4$. Let $\{x, y\}$ be a basis of $A$, with corresponding invariants $S=\bigoplus_{p \in P} \boldsymbol{Z}\left(p^{k(p)}\right), c \in \hat{\boldsymbol{Z}}$ as in Corollary 1.

(a) $t(x) \wedge t(y)$ is a quasi-isomorphism invariant of $A$, henceforth denoted $t_{0}$.

(b) $t(x)+t(y)+t(k)$ is a quasi-isomorphism invariant of $A$, henceforth denoted $s_{0}$.

(c) For any $z \in A$, define $\chi(z) \in \hat{\boldsymbol{Z}}$ by $\chi(z)(p)=p^{h(z)(p)}$, (interpreted as zero if $h(z)(p)=\infty)$. Let $\left\{x^{\prime}, y^{\prime}\right\}$ be a basis of $A$ with $x^{\prime}=r x+$ $s y, y^{\prime}=r^{\prime} x+s^{\prime} y$, where $r, s, r^{\prime}, s^{\prime} \in \boldsymbol{Q}$. Let $c^{\prime} \in \hat{\boldsymbol{Z}}$ be the corresponding invariant as in Corollary 1. Then

$$
t\left(\chi\left(y^{\prime}\right) c^{\prime}(s \chi(y) c-r \chi(x))+\chi\left(x^{\prime}\right)\left(s^{\prime} \chi(y) c-r^{\prime} \chi(x)\right) \geqq s_{0} .\right.
$$


CoRollary 2. The irrational $c(p)$ 's defined in Corollary 1 are a quasi-isomorphism invariant of $A$; that is, if c corresponds to a basis $\{x, y\}$ and $c^{\prime}$ to $\left\{x^{\prime}, y^{\prime}\right\}$, then for any $p \in P, c(p)$ is irrational iff $c^{\prime}(p)$ is irrational.

Proof. Let $k, k^{\prime}$ be the heights corresponding to $\{x, y\},\left\{x^{\prime}, y^{\prime}\right\}$. (See Proposition 1 for notation.) If $s_{0}(p)$ is finite, then $k(p), k^{\prime}(p)$ are both finite so $c(p), c^{\prime}(p)$ are necessarily rational.

Assume then that $s_{0}(p)=\infty$, and $c^{\prime}(p)$ is irrational. This implies that $k^{\prime}(p)=\infty$, and that $u^{\prime}=h\left(y^{\prime}\right)(p)$ and $v^{\prime}=h\left(x^{\prime}\right)(p)$ are finite. Let $u=h(y)(p), v=h(x)(p)$; by Proposition $1(\mathrm{c})$,

$$
t\left(\left(p^{u^{\prime}} c^{\prime}(p)\left(s p^{u} c(p)-r p^{v}\right)+p^{v^{\prime}}\left(s^{\prime} p^{u} c(p)-r^{\prime} p^{v}\right)\right)(p)=\infty,\right.
$$

so $p^{u^{\prime}} c^{\prime}(p)\left(s p^{u} c(p)-r p^{v}\right)=-p^{v^{\prime}}\left(s^{\prime} p^{u} c(p)-r^{\prime} p^{v}\right)$.

If $u=\infty$, then $p^{u^{\prime}+v} r c^{\prime}(p)=p^{v^{\prime}+v} r^{\prime}$, which is rational, so $v=\infty$. But then every element of $A$ has infinite $p$-height, contradicting the finiteness of $u^{\prime}$, so $u$ and similarly $v$ are finite. Hence $c(p) \neq 0$ and $k(p)=\infty$.

Now $s p^{u} c(p)-r p^{v}=0$ iff $s^{\prime} p^{u} c(p)-r^{\prime} p^{v}=0$, contradicting the linear independence of $\left\{x^{\prime}, y^{\prime}\right\}$, so neither are zero and

$$
c^{\prime}(p)=-p^{v^{\prime}-u^{\prime}}\left(s^{\prime} p^{u} c(p)-r^{\prime} p^{v}\right) /\left(s p^{u} c(p)-r p^{v}\right) .
$$

Since $c^{\prime}(p)$ is irrational, so is $c(p)$. Reversing the roles of $c(p)$ and $c^{\prime}(p)$ throughout yields a proof that if $c(p)$ is irrational, so is $c^{\prime}(p)$.

We now use Proposition 1 and Corollary 2 to identify certain sets of primes which are quasi-isomorphism invariants of $A$.

A prime $p$ is called accidental if $s_{0}(p)=\infty>t_{0}(p)$. An accidental prime $p$ is flat if for any choice of basis, $c(p)$ is always irrational; it is sharp otherwise. Note that Corollary 2 implies that the flat primes are a quasi-isomorphism invariant of $A$; the sharp primes are not: for example, if $p$ is sharp for some choice $\{x, y\}$ of basis, then there is an element $z$ with $h(z)(p)=\infty$, and $\{x, z\}$ is a basis with respect to which $p$ is not even accidental.

We shall also need Lemma 9.1 and Corollary 7.4 of [1], which translated into the notation of Proposition 1 become:

Proposition 2. Let $\{x, y\}$ be a basis of $A$, and let $z=r x+s y \epsilon$ $A, r, s \in \boldsymbol{Q} . \quad$ Then $h(z)=\min \{h(s \chi(y) c-r \chi(x), k+h(y)+h(s), k+$ $h(x)+h(r)\}$.

CoROllaRY 3. $h(z)(p)=\infty$ iff $k(p)=\infty$ and $r / s=p^{h(y)(p)-h(x)(p)} c(p)$; in particular, if $h(z)(p)=\infty$, then $h(r / s)(p)=h(y)(p)-h(x)(p)$. 
Proposition 3. Let $\{x, y\}$ be a basis of $A$. Define $\rho \in \hat{Z}$ by

$$
\rho(p)=\left\{\begin{array}{l}
c(p) \text { if } h(x)(p)=h(y)(p), \\
0 \text { otherwise }
\end{array}\right.
$$

and

$$
\Delta: \hat{Z} \longrightarrow\{0, \infty\} \text { by } \Delta(\chi)(p)=\left\{\begin{array}{lll}
0 & \text { if } & \chi(p) \neq 0 \\
\infty & \text { if } & \chi(p)=0
\end{array}\right.
$$

Let $z \in A \backslash\{W(x) \cup W(y)\}$; then $t(z)=t(a x+b y)$ for some nonzero coprime pair $(a, b)$ of integers. Let $r=a / b$, and let

$$
t_{r}=t(k \wedge(h(r-\rho)+\Delta(r \chi(x)-c \chi(y)))) .
$$

Then $t(z)=t_{0}+t_{r}$, and $T(A)=\left\{t(x), t(y), t_{0}+t_{r}: 0 \neq r \in \boldsymbol{Q}\right\}$.

The following lemmas show how accidental primes affect the typeset of $A$.

Lemma 1. Let $p$ be a sharp prime. Then there exists $z \in A$ with $h(z)(p)=\infty$, but for all $w \notin W(z), h(w)(p)<\infty$.

Proof. For some choice $\{x, y\}$ of basis, $h(x)(p)$ and $h(y)(p)$ are finite, $k(p)=\infty$ and $c(p)=a / b$ for coprime nonzero integers $a, b$ each prime to $p$. Take $z=a \chi(y)(p) x+b \chi(x)(p) y \in A$. By Proposition 2, $h(z)(p)=\infty$.

If $w \notin W(z),\{w, z\}$ is basis and $t_{0}=t(w) \wedge t(z)$, so $h(w)(p)<\infty$.

Lemma 2. Let $p$ be flat prime. Then for all $0 \neq z \in A, h(z)(p)<$ $\infty$; for each basis $\{x, y\}$ of $A, k(p)=\infty$; there are infinitely many pairwise independent $z_{i}$ with $h\left(z_{i}\right)(p)<h\left(z_{j}\right)(p)$ whenever $i<j$.

Proof. Let $c$ be the invariant corresponding to any basis $\{x, y\}$. Since $c(p)$ is irrational, for all $z \neq 0, h(z)(p)<\infty$, by Proposition 2, and $k(p)=\infty$. For $j=1,2, \cdots$, define $z_{j}=c_{j}(p) \chi(y)(p) x+\chi(x)(p) y \in A$. Then

$$
\begin{aligned}
h\left(z_{j}\right)(p) & =h(x)(p)+h(y)(p)+h\left(c(p)-c_{j}(p)\right)(p) \\
& \geqq h(x)(p)+h(y)(p)+j .
\end{aligned}
$$

Hence there is a subsequence $\left(z_{i}\right)$ of the $\left(z_{j}\right)$ satisfying $h\left(z_{i}\right)(p)<$ $h\left(z_{j}\right)(p)$ if $i<j$. The $\left(z_{i}\right)$ are pairwise independent, for if $a z_{i}=b z_{j}$ for integers $a, b$ with $i<j$, then $\left(a c_{i}(p)-b c_{j}(p)\right) x=(b-a) y$, so $a=b$ and $z_{i}=z_{j}$, a contradiction. 
4. Admissible typeset-cotypeset pairs. For any group $A$, the typeset of $A, T(A)$, is the set of types of rank 1 pure subgroups of $A$, and the cotypeset of $A, T^{\prime}(A)$, is the set of types of rank 1 torsionfree factor groups of $A$. In case rank $A=2,\left(T(A), T^{\prime}(A)\right)$ is the set of pairs of types of the form $\left(t, t^{\prime}\right)$, where for some $0 \neq x \in A$, $t$ is the type of $W(x)$, and $t^{\prime}$ is the type of $A / W(x)$. A set $\left(T, T^{\prime}\right)$ of pairs of types is called admissible if for some rank 2 group $A,\left(T, T^{\prime}\right)=$ $\left(T(A), T^{\prime}(A)\right)$. The following necessary conditions for admissibility follow immediately from $\S \S 2$ and 3 .

Proposition 4. If $\left(T, T^{\prime}\right)$ is admissible, then:

(1) $\left|\left(T, T^{\prime}\right)\right|$ is finite or countable.

(2) There is a type $t_{0}$ such that, for all $t_{1} \neq t_{2}$ in $T, t_{1} \wedge t_{2}=t_{0}$.

(3) There is a type $s_{0}$ such that, for all $\left(t, t^{\prime}\right) \in\left(T, T^{\prime}\right), t+t^{\prime}=s_{0}$.

(4) If $\left(t_{1}, t_{1}^{\prime}\right) \neq\left(t_{2}, t_{2}^{\prime}\right) \in\left(T, T^{\prime}\right)$, then $t_{1} \leqq t_{2}^{\prime}$. If $\left(T, T^{\prime}\right)=\left\{\left(t, t^{\prime}\right)\right\}$, then $t \leqq t^{\prime}$.

We now wish to show that the conditions of Proposition 4 are also sufficient for admissibility; we can make the computations less onerous by means of the following lemma, which allows us to assume $t_{0}=(\boldsymbol{Z})$.

LEMMA 3. Given $\langle A, W(x), W(y), \bar{W}(x), \bar{W}(y), S ;[\beta, \gamma]\rangle$, let $h_{0}=$ $h(x) \wedge h(y)$; let $G$ be that subgroup of $\boldsymbol{Q}$ containing 1 in which $h(1)=h_{0}$. Let $A^{\prime}$ be that subgroup of $V$ containing $\{x, y\}$ in which $h_{A^{\prime}}(x)=h_{A}(x)-h_{0}$ and $h_{A^{\prime}}(y)=h_{A}(y)-h_{0}$, but otherwise $A^{\prime}$ has the same invariants $S$ and $[\beta, \gamma]$ as $A$.

Then $A \cong G \otimes A^{\prime}$, the invariant $t_{0}^{\prime}$ of $A^{\prime}$ is $t(Z)$, and $T(A)=$ $\left\{t+t_{0}: t \in T\left(A^{\prime}\right)\right\}$.

Proof. There is a canonical injection $A^{\prime} \rightarrow G \otimes A^{\prime}$ such that $h_{G \otimes A^{\prime}}(1 \otimes x)=h_{G}(1)+h_{A^{\prime}}(x)=h_{A}(x)$, and $h_{G \otimes A^{\prime}}(1 \otimes y)=h_{A}(y)$.

Let $W^{\prime}(x), W^{\prime}(y)$ be the pure subgroups of $A^{\prime}$ generated by $x$ and $y$, and let $\bar{W}^{\prime}(x) \cong A^{\prime} / W^{\prime}(x), \bar{W}^{\prime}(y) \cong A^{\prime} / W^{\prime}(y)$ be the corresponding complementary subgroups of $V$, as in $\S 2$. Since $h_{\bar{W}(y)}(x)=h_{G}(1)+$ $h_{\bar{W}^{\prime}(y)}(x), h_{\bar{W}(x)}(y)=h_{G}(1)+h_{\bar{W}^{\prime}(x)}(y)$, and $G \otimes S \cong \bigoplus_{p \in P}\left(G \otimes Z\left(p^{k(p)}\right)\right) \cong$ $S$, there is an exact commutative diagram derived from $\S 2$, in which the unlabelled oblique arrows represent isomorphisms: 


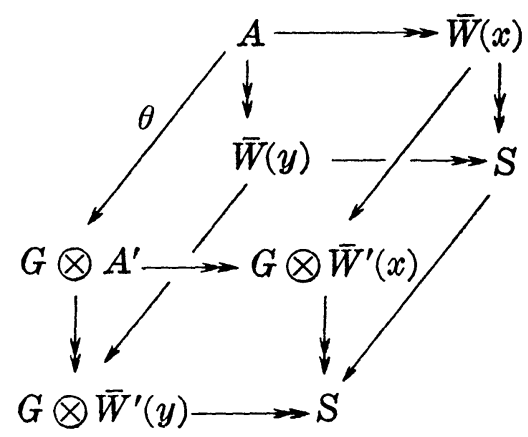

Hence $\theta$ is also an isomorphism. Then the statements about $t_{0}^{\prime}$ and $T\left(A^{\prime}\right)$ follow from Proposition 2.

5. The construction. Let $\left(T, T^{\prime}\right)$ be a set satisfying conditions (1)-(4) of Proposition 4. By Lemma 3, we shall assume that for all $t \neq t^{\prime} \in T, t \wedge t^{\prime}=t(\boldsymbol{Z})$; (or, if $T=\{t\}$, that $t=t(\boldsymbol{Z})$ ). Let $0=h_{0} \in$ $t_{0}=t(\boldsymbol{Z})$; choose any $\left(t_{1}, t_{1}^{\prime}\right) \in\left(T, T^{\prime}\right)$ and let $s_{0}=t_{1}+t_{1}^{\prime}$. Choose $h_{0}^{\prime} \in s_{0}$, and let $h_{1} \in t_{1}$ such that $h_{1} \leqq h_{0}^{\prime}$. Let $h_{1}^{\prime}=h_{0}^{\prime}-h_{1} \in t_{1}^{\prime}$ (where we take $\infty-\infty=0)$.

If $\left(T, T^{\prime}\right)=\left\{\left(t_{1}, t_{1}^{\prime}\right)\right\}$, let $\left(h_{2}, h_{2}^{\prime}\right)=\left(h_{1}, h_{1}^{\prime}\right)$ and $k=h_{1}^{\prime}-h_{1}$. Otherwise, choose $\left(t_{2}, t_{2}^{\prime}\right) \neq\left(t_{1}, t_{1}^{\prime}\right)$ from $\left(T, T^{\prime}\right)$. Since $t_{1} \wedge t_{2}=t_{0}$ and $t_{2} \leqq t_{1}^{\prime}$, for any $h \in t_{2},\left(\left\{p: 0<h_{1}(p)\right\} \cap\{p: 0<h(p)\}\right) \cup\left\{p: h_{1}^{\prime}(p)<h(p)\right\}$ is finite, so there exists $h_{2} \in t_{2}$ such that $h_{2} \leqq h_{1}^{\prime}$, and for all $p$ such that $0<h_{1}(p)$, $h_{2}(p)=0$ or $\infty$. Let $k=h_{1}^{\prime}-h_{2}$, and let $h_{2}^{\prime}=h_{1}+k \in t_{2}^{\prime}$. From $V$ take an independent pair $\{x, y\}$, and set $h(x)=h_{1}, h(y)=h_{2}$; we have now established our cadre $\left\langle W(x), W(y), \bar{W}(x), \bar{W}(y), S=\bigoplus_{p \in P} \boldsymbol{Z}\left(p^{k(p)}\right)\right\rangle$ as in $\S 2$. In order to construct $A$ realizing $\left(T, T^{\prime}\right)$, it remains to find a suitable $c(p)$ for each prime $p$. In the process, we shall also find for each pair $\left(t, t^{\prime}\right)$ a rational $r=a / b$ such that $z=a x+b y \in A$ and $t(z)=t, t(A / W(Z))=t^{\prime}$.

Let $\left(t_{i}\right)$ be any ordering of $T$; note that for all $i \neq 1,2, t_{i} \leqq t_{1}^{\prime}=$ $t\left(h_{2}+k\right)$ and $t_{i} \leqq t\left(h_{1}+k\right)$, so $t_{i} \leqq t_{0}+t(k)$. Assume that for all $j$, $3 \leqq j<i$, we have found $\left(h_{j}, h_{j}^{\prime}\right) \in\left(t_{j}, t_{j}^{\prime}\right)$ satisfying $\forall p \in \bigcup_{l<j}\{p: 0<$ $\left.h_{l}(p)\right\}, h_{j}(p)=0$ or $\infty$, and for all $l<j, h_{j} \leqq h_{l}^{\prime}$ and $h_{j} \leqq k$. If $\left(T, T^{\prime}\right)$ is exhausted, we are done; otherwise, for any $h \in t_{i},\{p: 0<h(p)\} \cap$ $\left(\bigcup_{j<i}\left\{p: 0<h_{j}^{\prime}(p)\right\}\right) \cup \bigcup_{j<i}\left\{p: h_{j}^{\prime}(p)_{i}^{\prime}<h(p)\right\} \cup\{p: h(p)>k(p)\}$ is finite, so there exists $h_{i} \in t_{i}$ such that, for all $j<i, h_{i} \leqq h_{j}^{\prime}, h_{i} \leqq k$, and for all $p \in \mathbf{U}_{j<i}\left\{p: 0<h_{i}(p)\right\}, h_{i}(p)=0$ or $\infty$. Let $h_{i}^{\prime}=h_{0}^{\prime}-h_{i} \in t_{i}^{\prime}$. By induction, we have found, for all $i,\left(h_{i}, h_{i}^{\prime}\right) \in\left(t_{i}, t_{i}^{\prime}\right)$ satisfying

(1) $\forall j<i, h_{i} \leqq h_{j}^{\prime}$

(2) $\forall p \in \bigcup_{j<i}\left\{p: 0<h_{j}(p)\right\}, h_{i}(p)=0$ or $\infty$

and

(3) $h_{i} \leqq k$. 
Having found suitable $\left(h_{i}, h_{i}^{\prime}\right)$ for all $i$, we proceed to partition $P$ with respect to these heights.

Let $S(0)=\{p: k(p)=0\}$, and $S^{\prime}(0)=\left\{p: k(p) \neq 0\right.$ but $\left.\forall i, h_{i}(p)=0\right\}$. For $i \geqq 1$, let $S(i)=\left\{p: 0<h_{i}(p)<\infty\right\}$, and $R(i)=\left\{p: h_{i}(p)=\infty\right\}$. Note that the $S(i)$ and $S^{\prime}(0)$ are disjoint for all $i \geqq 0$; by Lemma 1 , $R=\bigcup_{i} R(i)$ is the set of sharp primes, and the set of flat primes is $\{p: k(p)=\infty$ but $p \notin R\}$. Furthermore, $\boldsymbol{P}=\bigcup_{i \geqq 0}(S(i) \cup R(i)) \cup S^{\prime}(0)$, and $R(1) \cup R(2) \subseteq S(0)$.

For $i \geqq 1$, let $U(i)=\bigcup_{j<i}(S(j) \cap R(i))$, so the $U(i)$ are finite and disjoint, perhaps empty.

Next, we choose rationals $r$ such that $T=\left\{t_{1}, t_{2}, t_{r}: 0 \neq r \in \boldsymbol{Q}\right\}$ as in Proposition 3. Suppose that for all $j$ with $3 \leqq j<i$, distinct $r_{j}$ have been chosen satisfying:

$\left(j_{1}\right) \quad \forall p \in R(j), h\left(r_{j}\right)(p)=h(y)(p)-h(x)(p)$

$\left(j_{2}\right) \quad \forall q \in \bigcup_{k<j} U(k), h\left(r_{j}\right)(q) \neq h(y)(q)-h(x)(q)$.

Now choose $r_{i}$ different from all previously selected $r_{j}$ satisfying $\left(i_{1}\right)$ and $\left(i_{2}\right)$. Such a choice is always possible in $\aleph_{0}$ different ways, since

(a) $\forall p \in R(i), h(y)(p)=0=h(x)(p)$ except for $p$ in the finite set $X=(S(1) \cup S(2)) \cap R(i)$,

(b) $\bigcup_{j<i} U(j)$ is finite and disjoint from $R(i)$, and

(c) if $R(i)=\boldsymbol{P}$, then $A$ is not reduced.

For example, let $m$ be an integer not in $R(i)$ such that

$$
\forall q \in \bigcup_{j<i} U(j), h(m)(q)>h(y)(q)-h(x)(q),
$$

and let $n=\prod_{p \in X} p^{h(y)(p)-h(x)(p)}$. For any positive integer $a$, let $s^{a}=$ $m^{a} n$; then $\left\{s^{a}: a=1,2, \cdots\right\}$ is an infinite set of integers satisfying $\left(i_{1}\right)$ and $\left(i_{2}\right)$ of which at most finitely many have previously been chosen as $r_{j}^{\prime}$ 's.

By induction, we have defined a $1-1$ function $t_{i} \mapsto r_{i}$ for all $i \geqq 3$ such that for all $i, r_{i}$ satisfies $\left(i_{1}\right)$ and $\left(i_{2}\right)$. Furthermore, we have shown that if $T$ is finite, such a function can be chosen in $\boldsymbol{\aleph}_{0}$ ways, while if $T$ is infinite, it can be chosen in $2^{N_{0}}$ ways.

We now assign values to the $c(p)$. For $p \in S(0)$, let $c(p)=0$.

For $p \in S^{\prime}(0)$, let $c^{\prime}(p)$ be an integer prime to $p$ such that $0<$ $c^{\prime}(p)<p^{k(p)}$, and let $c(p)=c^{\prime}(p)+p u$, where $u=0$ if $k(p)<\infty$, and $u$ is an arbitrary irrational $p$-adic unit if $k(p)=\infty$. Let these $c(p)$ be chosen to be distinct, which is possible in $2^{\aleph_{0}}$ ways if at least one $k(p)=\infty$, in infinitely many ways if $S^{\prime}(0)$ is infinite, and otherwise in finitely many ways.

For $p \in R(i)$, let $c(p)=p^{h(x)(p)-h(y)(p)} r_{i}=p^{-h\left(r_{i}\right)(p)} r_{i}$, a rational $p$-adic unit. Because of conditions $\left(i_{2}\right)$ and $\left(j_{2}\right)$, if $p \in R(i)$ and $q \in R(j)$ with $i \neq j$, then $c(p) \neq c(q)$.

For $p \in S(i) \backslash R$, let $c^{\prime}(p)$ be an integer prime to $p$ such that $0<$ 
$c^{\prime}(p)<p^{k(p)}$, and, in case $i \geqq 3, c^{\prime}(p) \equiv p^{-h\left(r_{i}\right)(p)} r_{i}\left(\bmod p^{h_{i}(p)}\right)$; since $h_{i}(p) \leqq k(p)$, these conditions can be fulfilled. Let $c(p)=c^{\prime}(p)$ if $k(p)<$ $\infty$, and $c(p)=c^{\prime}(p)+u p$, where $u$ is any irrational $p$-adic unit, if $k(p)=\infty$. Note that for $p \neq q \in \mathrm{U}_{i} S(i) \backslash R$, it is not necessary to require $c(p) \neq c(q)$.

We have now assigned, for each prime $p$, a $p$-adic unit $c(p)$ such that

(1) $\forall i, \forall p \in R(i), c(p)=p^{-h\left(r_{i}\right)(p)} r_{i}$

(2) $\forall i, \forall p \in S(i), c(p) \equiv p^{-h\left(r_{i}\right)(p)} r_{i}\left(\bmod p^{h_{i}(p)}\right)$

(3) if $p$ is flat, $c(p)$ is irrational; otherwise $c(p)$ is rational. Thus we have all the data required to construct $A$.

6. Proof of admissibility of $\left(T, T^{\prime}\right)$. To show that $A$ realizes $\left(T, T^{\prime}\right)$, we shall use Proposition 3 in the form $T(A)=\{t(x), t(y)$, $\left.t_{r}: 0 \neq r \in \boldsymbol{Q}\right\}$. For any $0 \neq r \in \boldsymbol{Q}$, let $h_{r}$ be the generalized height defined by:

$$
h_{r}(p)=\min \{k(p), h(r-\rho(p))+\Delta(r \chi(x)(p)-c(p) \chi(y)(p))\},
$$

so $t_{r}=t\left(h_{r}\right)$.

Firstly, suppose $r=r_{i}$ for some $i$. Then:

(1) For $p \in S(0), h_{r}(p)=h_{i}(p)=0$.

(2) For $p \in S^{\prime}(0)$, either $c(p)=r$ which can happen for at most one $p$, and $h_{r}(p)=k(p)$, or $c(p) \neq r$, in which case $h_{r}(p)=\min \{k(p)$, $h(r)(p)\}$. Now if $p \in S^{\prime}(0)$ and $k(p)=\infty$, then $p$ is flat, so $c(p) \neq r$; hence in either case, $h_{r}(p)$ is finite and zero except for finitely many primes.

(3) For $p \in U(i), k(p)=\infty$ and $r \chi(x)(p)=c(p) \chi(y)(p)$, so $h_{r}(p)=$ $\infty=h_{i}(p)$.

(4) For $p \in R(i) \backslash U(i), k(p)=\infty, \chi(x)(p)=\chi(y)(p)=1$ and $r=$ $c(p)$, so $h_{r}(p)=\infty=h_{i}(p)$.

(5) For $p \in R(j), i \neq j, k(p)=\infty$ and $c(p)=r_{j} \neq r_{i}$, so $r-\rho(p)$ is a nonzero $p$-adic integer while $\Delta(r \chi(x)(p)-c(p) \chi(y)(p))=0$. Hence $h_{r}(p)=h_{i}(p)=0$ except for finitely many primes where both are finite.

(6) For $p \in S(1) \cup S(2) \backslash R, 0<k(p)<\infty$ and either $c(p) \neq$ $r p^{h(x)(p)-h(y)(p)}$, in which case $h_{r}(p)=\min \{k(p), h(r)(p)\}$, which is zero except for finitely many primes, or $c(p)=r p^{h(x)(p)-h(y)(p)}$, in which case $h_{r}(p)=k(p)$. But since $h(x)(p)>0$, or $h(y)(p)>0$, this can only happen for finitely many primes, so for all but finitely many primes, $h_{r}(p)=0=h_{i}(p)$.

(7) For $p \in S(i) \backslash R, i \geqq 3,0<k(p)<\infty$ and

$$
c(p) \equiv p^{-h\left(r_{i}\right)(p)} r_{i}\left(\bmod p^{h_{i}(p)}\right),
$$

while $h(x)(p)=h(y)(p)=0$. Either $h(r)(p)=0$ and $h_{r}(p)=\min \{k(p)$, $\left.h_{i}(p)\right\}=h_{i}(p)$, or $h(r)(p) \neq 0$, and $h(r)(p)$ is finite. But the latter can 
occur only for finitely many primes.

( 8 ) For $p \in S(j) \backslash R, j \geqq 3, h(x)(p)=h(y)(p)=0$, so $h_{r}(p)=$ $\min \{k(p), h(r-c(p))(p)+\Delta(r-c(p))(p)\}$. Since $r \neq c(p)$, this is finite and zero except for the finitely many primes $p$ for which $h(r-c(p))(p)>0$. Since $h_{\imath}(p)=0, h_{r}(p)=h_{\imath}(p)$ for almost all $p$.

We have considered all primes, and can conclude that $t_{r}=t_{i}$. Next, suppose $r \neq r_{i}$.

(1) For $p \in S(0), h_{r}(p)=h_{i}(p)=0$.

(2) For $p \in S^{\prime}(0)$, just as in the case $r=r_{i}$, we have that $h_{r}(p)$ is finite and zero except for finitely many primes.

(3) For $p \in R(i), k(p)=\infty=h_{i}(p)$, but $r \chi(x)(p) \neq c(p) \chi(y)(p)$, so $h_{r}(p)=h(r)(p)$ or $h(r-c(p))(p)$, which is finite.

(4) For $p=S(i), h_{r}(p)=h_{i}(p)$ iff $p^{-h(r)(p)} r \equiv p^{-h\left(r_{i}\right)(p)} r_{\imath}\left(\bmod p^{h_{\imath}(p)}\right)$, but this can happen for only finitely many primes.

Hence $t_{r} \neq t_{i}$ unless both are $t_{0}$. We conclude that $t_{r}=t_{2}$ if and only if $r=r_{i}$ or $r \neq r_{j}$ for all $j$ and $t_{r}=t_{0}$.

Since the group $A$ so constructed has the height $k$ and the accidental primes completely determined by $\left(T, T^{\prime}\right)$, not only $T=T(A)$, but also $T^{\prime}(A)=T^{\prime}$. This answers Question 2(a) of [1].

7. Number of groups realizing an admissible $\left(T, T^{\prime}\right)$. We saw in $\S 3$ above that for fixed $W(x), W(y)$, and $k$, distinct classes $[\beta, \gamma]$ of epimorphisms produce nonisomorphic groups $A$. Each class $[\beta, \gamma]$ yields a distinct $c \in \hat{\boldsymbol{Z}}$, where $c(p)$ is unique modulo $p^{k(p)}$ if $k(p)<\infty$, and conversely if $A$ corresponds to $c \in \hat{Z}$, and $A^{\prime}$ to $c^{\prime}$, then $A$ is quasi-isomorphic to $A^{\prime}$ iff $c$ differs from $c^{\prime}$ by a rational multiple (modulo $p^{k(p)}$ if $\left.k(p)<\infty\right)$.

Thus, the number of groups realizing $\left(T, T^{\prime}\right)$ is equal to the number of possible choices of the $c(p)$, given a fixed ordering $\left(t_{i}\right)$ of $T$.

THEOREM 1. Let $\left(T, T^{\prime}\right)$ be a pair of sets of types satisfying conditions (1)-(4) of Proposition 4, and let $c\left(T, T^{\prime}\right)$ denote the number of isomorphism classes of rank 2 groups realizing $\left(T, T^{\prime}\right)$, and $c^{\prime}\left(T, T^{\prime}\right)$ the number of quasi-isomorphism classes. Then, in the notation of $\S 6$ :

(1) If $S=\bigoplus_{p \in P} \boldsymbol{Z}\left(p^{k(p)}\right)$ is finite, $c\left(T, T^{\prime}\right) \leqq$ number of units of $S$, considered as a unital cyclic ring and $c^{\prime}\left(T, T^{\prime}\right)=1$.

(2) If $S$ is infinite, there are no flat primes and $\{t \in T: \exists p$ with $t(p)=\infty\}$ is finite, then $c^{\prime}\left(T, T^{\prime}\right) \leqq \aleph_{0}=c\left(T, T^{\prime}\right)$.

(3) Otherwise, $c\left(T, T^{\prime}\right)=2^{\aleph_{0}}=c^{\prime}\left(T, T^{\prime}\right)$.

Proof. Since $A$ is a subset of a 2 dimensional rational vector space, we certainly have $c^{\prime}\left(T, T^{\prime}\right) \leqq c\left(T, T^{\prime}\right) \leqq 2^{\mathrm{N}_{0}}$. 
(1) In the construction of $\S 6$, we chose $c$ among the units of $S$. If $\alpha_{1}, \alpha_{2}$ are any automorphisms of $S, \alpha_{1}$ is a rational multiple of $\alpha_{2}$, so $c^{\prime}\left(T, T^{\prime}\right)=1$. In fact, each $A$ realizing $\left(T, T^{\prime}\right)$ is quasiisomorphic to $W(x) \oplus W(y)$.

(2) Let $H=\{i: R(i) \neq \varnothing\}$ be a finite set. For each $i \in H$, we chose $r_{i}$ from an infinite set of candidates, and each such choice determined a unique $c(p)$ for all $p \in R(i)$. The remaining $c(p)$ were each chosen from a finite set, so all in all, there were $\aleph_{0}$ possible choices for $c$, so $c\left(T, T^{\prime}\right)=\boldsymbol{\aleph}_{0}$.

(3) If any $p$ is flat, an arbitrary choice of an irrational $p$-adic unit was made in the construction, so $c\left(T, T^{\prime}\right)=2^{\aleph_{0}}$.

If $\{t: \exists p$ with $t(p)=\infty\}$ is infinite, an infinite number of choices of distinct $r_{i}$ were made, each from an infinite set, and each such choice defined a unique $c$, so $c\left(T, T^{\prime}\right)=2^{\aleph_{0}}$.

Of these $2^{\aleph_{0}}$ possible $c$, at most $\aleph_{0}$ can be related by a rational multiple, so $c^{\prime}\left(T, T^{\prime}\right)=2^{\aleph_{0}}$.

EXAMPLE. Classification of rank 2 homogeneous groups:

Let $\left(T(A), T^{\prime}(A)\right)=\left\{\left(t, t^{\prime}\right)\right\} ;$ by Lemma $3, A \cong G \otimes A^{\prime}$, where $G$ is a rank 1 group and $\left(T\left(A^{\prime}\right), T^{\prime}\left(A^{\prime}\right)\right)=\{(t(\boldsymbol{Z}), t(k))\}$. Let

$$
B=\{p: k(p)=\infty\} \subseteq S^{\prime}(0) .
$$

If $B \neq \varnothing$, there are $2^{\aleph_{0}}$ possibilities for the quasi-isomorphism class of $A^{\prime}$; if $B=\varnothing$ but $S^{\prime}(0)$ is infinite, there are $\boldsymbol{\aleph}_{0}$ possibilities for the quasi-isomorphism class of $A^{\prime}$; otherwise $A^{\prime} \doteq Z \oplus Z$.

8. Completely anisotropic groups. Beaumont and Pierce [1, Definition 7.8] define $A$ to be completely anisotropic (c.a.) if no two independent elements have the same type. They show that for any rank 2 group, the only type which can possibly be the type of two independent elements is $t_{0}$, and hence if $t_{0} \notin T(A)$, then $A$ is c.a.; furthermore, if $T(A)$ is finite, then $A$ cannot be c.a. They prove the existence of c.a. groups but do exhibit an example; indeed the first explicit example in the literature occurs in [5, Theorem 1], although Dubois [3, Theorem 1] gives a necessary condition for a typeset to be realized by a c.a. group, and both he and Koehler [6] exhibit an infinite admissible typeset which cannot be realized by a c.a. group. Ito [5] gives a sufficient, but not necessary condition for a typeset to be realized by a c.a. group.

The following proposition provides a necessary condition for a group to be c.a., and the next theorem proves that it is also sufficient.

Proposition 5. Let $A$ be a rank 2 group for which $t_{0}=t(\boldsymbol{Z})$. If $A$ is c.a., then for any basis $\{x, y\}$ with $h(x) \wedge h(y)=0$, there 
are infinitely many types $t$ in $T(A)$ satisfying:

$$
\forall p \text {, if } t(p)=\infty \text {, then } h(y)(p)-h(x)(p)=0 \text {. }
$$

Proof. Since $A$ is c.a., there is a prime $p$ with $h(y)(p) \neq h(x)(p)$. Let $r_{k}=p^{h(y)(p)-h(x)(p)+k}$, so $\left\{r_{k}: k=1,2, \cdots\right\}$ is an infinite set of rationals such that $h\left(r_{k}\right)(q) \neq h(y)(q)-h(x)(q)$ for all $q$ for which $h(y)(q)-$ $h(x)(q) \neq 0$. Let $r_{k}=a_{k} / b_{k}$, where $a_{k}, b_{k} \in \boldsymbol{Z}$.

By Corollary 3, the elements $z_{k}=a_{k} x+b_{k} y$ satisfy: if $h(y)(p)-$ $h(x)(p) \neq h\left(r_{k}(p)\right.$, then $h\left(z_{k}\right)(p) \neq \infty$. But since $A$ is c.a., the $z_{k}$, being pairwise independent, all have different types, so there are infinitely many types $t$ in $T(A)$ such that if $t(p)=\infty$, then $h(y)(p)-h(x)(p)=0$.

The following theorem provides a solution to Question $(2)(b)$ of [1]:

THEOREM 2. Let $\left(T, T^{\prime}\right)$ be a pair of sets of types satisfying conditions (1)-(4) of Proposition 4 (with $t_{0}=t(\boldsymbol{Z})$ ), and

(5) For any $t_{1}, t_{2} \in T$, let $h_{1} \in t_{1}, h_{2} \in t_{2}$. Then there are infinitely many $t \in T$ satisfying:

(c.a.) $\forall p$, if $t(p)=\infty$, then $h_{1}(p)-h_{2}(p)$ is finite, and zero almost everywhere.

Let $d\left(T, T^{\prime}\right)$ denote the number of isomorphism classes of c.a. rank 2 groups realizing $\left(T, T^{\prime}\right)$, and $d^{\prime}\left(T, T^{\prime}\right)$ the number of quasiisomorphism classes. Then:

(1) If there are no flat primes and $\{t \in T: \exists p$ with $t(p)=\infty\}$ is finite, then $d^{\prime}\left(T, T^{\prime}\right) \leqq \aleph_{0}=d\left(T, T^{\prime}\right)$.

(2) Otherwise $d^{\prime}\left(T, T^{\prime}\right)=2^{\aleph_{0}}=d\left(T, T^{\prime}\right)$.

Proof. In $\$ 6$, it was shown that for every nonzero rational $r, t_{r} \in T$ without repetitions iff the function $t_{i} \mapsto r_{i}$ in $\S 5$ is surjective. Hence by Theorem 1 above, it suffices to show that if condition (5) holds, we have the right number of surjective functions.

Let $L=\{t \in T: t$ satisfies (c.a.) $\}$, so $L$ is infinite. Let $M$ be the complement of $L$ in $T$, and order $T$ so that $M$ occurs before $L$.

We have seen in $\S 5$ that $\left\{r_{i}: t_{i} \in M\right\}$ can be chosen to leave an infinite complement in $\boldsymbol{Q}$. But then we have $\boldsymbol{\aleph}_{0}$ unused rationals and $\boldsymbol{\aleph}_{0}\left\{r_{i}, t_{i} \in L\right\}$ slots to fill, each of which is constrained by only finitely many conditions. Thus in case (1), we can choose $\aleph_{0}$ functions to be surjective, and in case (2), we can choose $2^{\aleph_{0}}$ surjective.

9. Acknowledgments. I gratefully acknowledge the hospitality 
of the Mathematics Department of the University of Washington, where this paper was written during my sabbatical leave.

\section{REFERENCES}

1. R. A. Beaumont and R. S. Pierce, Torsion-free groups of rank two, Mem. Amer. Math. Soc., 38 (1961).

2. D. W. Dubois, Applications of analytic number theory to the study of type sets of torsion-free Abelian groups I, Pub. Math., 12 (1965), 59-63.

3. - Applications of analytic number theory to the study of type sets of torsionfree Abelian groups II, Pub. Math., 13 (1966), 1-8.

4. L. Fuchs, Infinite Abelian Groups, Vol. I and II, Academic Press, New York and London, 1970, 1973.

5. R. Ito, On type-sets of torsion-free Abelian groups of rank 2, Proc. Amer. Math. Soc., 48, 1 (1975), 39-42.

6. J. Koehler, Some torsion-free rank two groups, Acta Sci. Math., 25, 1-2 (1964), 186-190.

7. P. Schultz, Torsion-free extensions of torsion-free Abelian groups, J. Algebra, 30 (1974), 75-91.

Received June 6, 1977 and in revised form November 1, 1977.

UNIVERSITY OF W. A.

Nedlands, Western Australia

Australia, 6009 



\title{
PACIFIC JOURNAL OF MATHEMATICS
}

\section{EDITORS}

RICHARD ARENS (Managing Editor)

University of California

Los Angeles, CA 90024

Charles W. Curtis

University of Oregon

Eugene, OR 97403

C. C. MOORE

University of California

Berkeley, CA 94720

\section{J. DUGUNDJI}

Department of Mathematics University of Southern California Los Angeles, CA 90007

R. Finn and J. Milgram

Stanford University

Stanford, CA 94305

\section{ASSOCIATE EDITORS}
E. F. BECKENBACH
B. H. NeumanN
F. WOLF
K. YOSHIDA

\section{SUPPORTING INSTITUTIONS}

\author{
UNIVERSITY OF BRITISH COLUMBIA \\ CALIFORNIA INSTITUTE OF TECHNOLOGY \\ UNIVERSITY OF CALIFORNIA \\ MONTANA STATE UNIVERSITY \\ UNIVERSITY OF NEVADA, RENO \\ NEW MEXICO STATE UNIVERSITY \\ OREGON STATE UNIVERSITY \\ UNIVERSITY OF OREGON
}

\author{
UNIVERSITY OF SOUTHERN CALIFORNIA \\ STANFORD UNIVERSITY \\ UNIVERSITY OF HAWAII \\ UNIVERSITY OF TOKYO \\ UNIVERSITY OF UTAH \\ WASHINGTON STATE UNIVERSITY \\ UNIVERSITY OF WASHINGTON
}

The Supporting Institutions listed above contribute to the cost of publication of this Journal, but they are not owners or publishers and have no responsibility for its content or policies.

Mathematical papers intended for publication in the Pacific Journal of Mathematics should be in typed form or offset-reproduced, (not dittoed), double spaced with large margins. Please do not use built up fractions in the text of the manuscript. However, you may use them in the displayed equations. Underline Greek letters in red, German in green, and script in blue. The first paragraph or two must be capable of being used separately as a synopsis of the entire paper. Items of the bibliography should not be cited there unless absolutely necessary, in which case they must be identified by author and journal, rather than by item number. Manuscripts, in triplicate, may be sent to any one of the editors. Please classify according to the scheme of Math. Reviews, Index to Vol. 39. All other communications should be addressed to the managing editor, or Elaine Barth, University of California, Los Angeles, California, 90024.

50 reprints to each author are provided free for each article, only if page charges have been substantially paid. Additional copies may be obtained at cost in multiples of 50 .

The Pacific Journal of Mathematics is issued monthly as of January 1966. Regular subscription rate: $\$ 72.00$ a year (6 Vols., 12 issues). Special rate: $\$ 36.00$ a year to individual members of supporting institutions.

Subscriptions, orders for numbers issued in the last three calendar years, and changes of address should be sent to Pacific Journal of Mathematics, P.O. Box 969, Carmel Valley, CA 93924, U.S.A. Older back numbers obtainable from Kraus Periodicals Co., Route 100, Millwood, NY 10546.

PUBLISHED BY PACIFIC JOURNAL OF MATHEMATICS, A NON-PROFIT CORPORATION

Printed at Kokusai Bunken Insatsusha (International Academic Printing Co., Ltd.). 8-8, 3-chome, Takadanobaba, Shinjuku-ku, Tokyo 160, Japan.

Copyright (C) 1978 by Pacific Journal of Mathematics Manufactured and first issued in Japan 


\section{Pacific Journal of Mathematics \\ Vol. 78, No. $2 \quad$ April, 1978}

Su-Shing Chen, Weak rigidity of compact negatively curved manifolds .... 273

Heinz Otto Cordes and D. A. Williams, An algebra of pseudodifferential operators with nonsmooth symbol ....................... 279

Herbert Paul Halpern, Normal expectations and integral decomposition of type III von Neumann algebras ......................... 291

G. Hochschild, On representing analytic groups with their

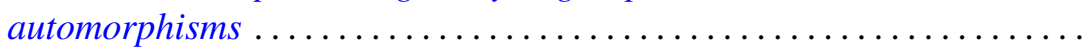

Dean G. Hoffman and David Anthony Klarner, Sets of integers closed under affine operators - the closure of finite sets ....................

Simeon Ivanov, On holomorphic relative inverses of operator-valued

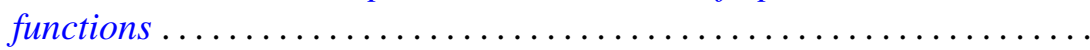

O. P. Juneja and M. L. Mogra, Radii of convexity for certain classes of

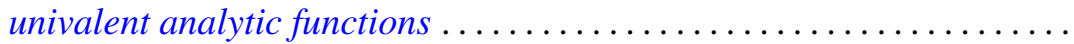

Hadi Kharaghani, The evolution of bounded linear functionals with application to invariant means.......................... 369

Jack W. Macki, A singular nonlinear boundary value problem .......... 375

A. W. Mason and Walter Wilson Stothers, Remarks on a theorem of $L$. Greenberg on the modular group ........................ 385

Kevin Mor McCrimmon, Peirce ideals in Jordan algebras . . . . . . . . . . . 397

John C. Morgan, II, On the absolute Baire property ................ 415

Gerard J. Murphy, Commutative non-Archimedean $C^{*}$-algebras ...

Masafumi Okumura, Submanifolds with L-flat normal connection of the

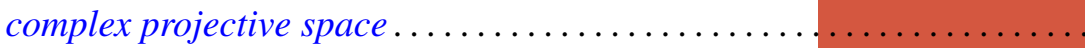

Chull Park and David Lee Skoug, Distribution estimates of barrier-crossing probabilities of the Yeh-Wiener process ...............

Irving Reiner, Invariants of integral representations ........

Phillip Schultz, The typeset and cotypeset of a rank 2 abelian group ..... 503

John Brendan Sullivan, Representations of Witt groups ....

Chia-Chi Tung, Equidistribution theory in higher dimensions . . . 